\title{
Augmenting Human Digital Memories with Physiological Data
}

\author{
Chelsea Dobbins, Madjid Merabti, Paul Fergus, David Llewellyn-Jones \\ School of Computing and Mathematical Sciences \\ Liverpool John Moores University \\ Liverpool, UK \\ C.M.Dobbins@2006.ljmu.ac.uk, \{M.Merabti, P.Fergus, D.Llewellyn-Jones \} @1jmu.ac.uk
}

\begin{abstract}
The area of human digital memories focuses on documenting our entire lifetime. Using this outlet, a diverse range of information can be brought together, such as photos, location, temperature and physiological information. Nowadays, we have access to a number of different data sources, thus allowing more dynamic and data rich memories to be created. In particular, the inclusion of physiological data offers a new insight into the augmentation of memories and provides a richer level of detail. This information can be used to determine how we were feeling, at any time, and, potentially, how we made others feel as well. Memories, created over a lifetime, can be retrieved, and we can see how our bodies have changed over time. This paper presents the DigMem system, which incorporates physiological data into the creation of human digital memories. A prototype has been successfully developed, which demonstrates the approach and evaluates the applicability of the research.
\end{abstract}

Keywords-Human Digital Memory, Lifelogging, Body Sensors, Physiological Monitoring, Mobile Devices

\section{INTRODUCTION}

Memories shape our existence. They link our past with our future and are composed of a variety of information. Supporting our memory, through cave paintings, storytelling, books and personal diaries, has been done for thousands of years and has been a way to preserve memories over a lifetime [1]. However, with the turn of the $21^{\text {st }}$ century there has been a fundamental advancement in technology. Computing devices are, nowadays, capable of capturing a multitude of personal data and can store a phenomenal amount of information. Our digital artefacts, or "memories," e.g. photos, videos, emails etc. are now the most popular representation of ourselves, and are the items that we hold onto and reflect over. Managing and using these digital memories, over a lifetime, has led to "Memories for Life" being declared a computing grand challenge [2].

The area of human digital memories (HDMs) focuses on documenting our entire lives. From the things we do, to the places we go, our interactions with others and even the thoughts we think. This outlet allows us to capture, from a variety of data sources, rich information about ourselves and our surrounding environment. HDMs are a representation of ourselves and evolve and grow alongside us. Therefore, simply arranging photos, videos or documents into a memory is not an accurate reflection of ourselves. The changes that our bodies were going through should also be incorporated into a HDM. This data is highly personalized and unique to every individual. Incorporating this data allows us to reflect on how we were feeling at any stage of our lives.

The influx of sensor and mobile devices, within our environment, has revolutionised the way in which a user can be monitored. Body sensors allow us to continuously monitor our person without too much intervention [3]. These devices were primarily used within wearable healthmonitoring systems (WHMS). However, as their popularity increases, they are branching out from the medical field and are increasingly being used within other domains. One such area is in the field of HDMs. Incorporating physiological data, from WHMS, provides vital information about ourselves and enables a richer understanding about our health, level of activity and physical wellbeing to emerge. By incorporating this information, along with a variety of other pieces of data, allows for a more dynamic memory to be created.

Sensor nodes and mobile phones provide an ideal, and unobtrusive, platform to obtain HDM data. Recent advancements in this field have enabled us to capture a multitude of data, on these instruments. Their ubiquitous nature and relatively small size allows them to be easily integrated into our environment. They now seamlessly fit into our world, instead of forcing us to enter the their environment, a concept first envisioned by Weiser [4]. Memories are impulsive and momentary and the data which embodies them is better suited to being captured on these portable devices and without too much user intervention.

This paper examines how physiological information can be used to augment a HDM. The DigMem system is presented. This system creates HDMs using a variety of information, obtained from pervasive devices. This includes information from physiological sensors, cameras and location data. Linked data is then used to bring together these fragmented pieces of information and create a memory box. In this context, a memory box is a temporal abstraction of a memory. All items from a specific time are brought together, into the "box," and a representation of that memory is presented. Memory boxes can be used to reason over behaviour from anytime in our lives and are able to provide a richer understanding about aspects of our health, level of activity and physical wellbeing.

\section{CAPTURING MEMORY DATA}


A plethora of information can be recorded about us and our environment. Automatically recording this data, and quantifying how a given aspect of our body changes over time, provides an insight into our underlying behaviours and is known as "lifelogging" [3], [5]. The content that is incorporated into lifelogs, or HDMs, is shifting from merely photos and videos to more sophisticated pieces of data, such as biological signals.

A revolutionary device, which has dominated the area of lifelogging, has been Microsoft's SenseCam [6]. This wearable device automatically triggers to capture photos, and is capable of storing up to 30,000 images. It contains a digital camera and multiple sensors, including sensors to detect changes in light levels, an accelerometer to detect motion, a thermometer to detect ambient temperature, and a passive infrared sensor to detect the presence of people [7]. The SenseCam has been used in various studies [5], [7-14] to monitor behaviour and capture memories. For example, Lee et al. [7] use the technology as a memory aid to capture the user's daily routine. The images recorded are then presented in a timeline format, similar to the approach used in Microsoft's MyLifeBits [15] project.

Whilst the methods and technologies discussed are a useful starting point novel solutions are needed, which include much richer data capturing capabilities. A less obtrusive and inexpensive method is required. In terms of capturing data, mobile phones fit well with our natural ability to move within our environment. Memories are not isolated static events, but rather a continuous sequence of experiences contextually linked and created within and across different geographical areas within the environments we occupy. This will be a key requirement in future work, along with building additional middleware services, to achieve the memory structures required. In this way, the services scheme provides a plug-and-play platform for memory data sources, which can be exploited by any digital life memory middleware services.

\section{PhysiologicAl MONITORING}

Sensor-based systems are quickly emerging as a new way to capture our every move and to monitor our health and wellbeing. These devices, particularly the one's used in WHMS, offer a new generation of inexpensive, unobtrusive wearable/implanted devices [3], which are capable of capturing content over a lifetime. However, these systems are mainly used within the area of healthcare. Nevertheless, a great deal can be learnt from their deployment in this field.

One such system that is being used to collect physiological data is the SenseWear Armband (SWA) [16]. This device collects data from a bi-axial accelerometer, galvanic skin resistance (sweat rates), heat flux (heat dissipated from the body), and skin and near body temperature, to estimate energy expenditure (EE) and step count [17]. The $S W A$ has been used to monitor adherence in women with rheumatoid arthritis, in a similar way to measuring decreases in sedentary behaviour [18]. The results from this study concluded with an $89 \%$ adherence rate and that the $S W A$ is a viable method of quantifying physical activity and may be useful to monitor effectiveness of interventions to increase activity in people with rheumatoid arthritis [18]. In relation to creating memories, this system can also be used to illustrate the movements of a user throughout the day. However, the context in which those movements occurred is unknown, without the use of a visual aid.

Whilst armbands are capable of measuring levels of physical activity, and energy expenditure, more sophisticated sensors are required to measure body signals. A number of wearable systems [19-28] have been designed for the purpose of measuring a user's physiological signals. One such approach has been Matthews et al.'s [29] "Physiological Sensor Suite (PSS)". This wearable device gathers electrocardiogram (ECG), electromyogram (EMG), electrooculogram (EOG) and through-hair electroencephalogram (EEG) data [29]. The information is then communicated, wirelessly, to a data logger, which the user is wearing. For the purpose of gathering information, this suite is particularly interesting because the sensors are quite small and "no modification of the skin's outer layer is required" [29]. In other words, the user doesn't have to prepare their skin with special gel (as is common practise amongst other systems).

As it can be seen, the use of sensor technology to measure physiological signals is mostly positive. However, in order to integrate this data into HDMs, advanced solutions are needed. This paper expands upon the technologies and ideas, described above, in order to integrate physiological signals and lifelogging data into HDMs. A vivid and interactive snapshot of our lives can be captured, reasoned upon and searched through. Interaction with these memories is fundamental to the idea and is what makes it unique. By enabling users to "go into" their memories and to see various information, such as temperature, location and emotions, could lead to the augmentation of group memories and has the advantage of benefiting numerous aspects of people's lives. Whether it enhances social groups and interactions or aids in the health and recovery of memory-related illness, the possibilities are endless.

\section{ThE DigMEM SYSTEM}

As it can be seen, many approaches exist that are capable of lifelogging and capturing physiological data. However, the domain's in which they are used are very separate. Capturing HDMs usually involves using specialist equipment (e.g. SenseCam) to record visual and locationspecific data, whilst the healthcare field use WHMS's to measure physiological information, for the purpose of developing personalised care. In order to form a betterrounded snapshot of our lives these technologies need to work together, so that we can visually recap our experiences, as well as the feelings and changes our bodies were going through when these events were occurring.

The DigMem system is composed of two applications Mobile DigMem (MoDM) and DigMem (see Fig. 1). The MoDM aspect gathers a variety of data from distributed sources, including photos, location-specific and physiological data. 
C. Dobbins, M. Merabti, P. Fergus, and D. Llewellyn-Jones, "Augmenting Human Digital Memories with Physiological Data," in The 3rd IEEE International Conference on Network Embedded Systems for Every Application (NESEA'12), 2012, pp. 35-41.

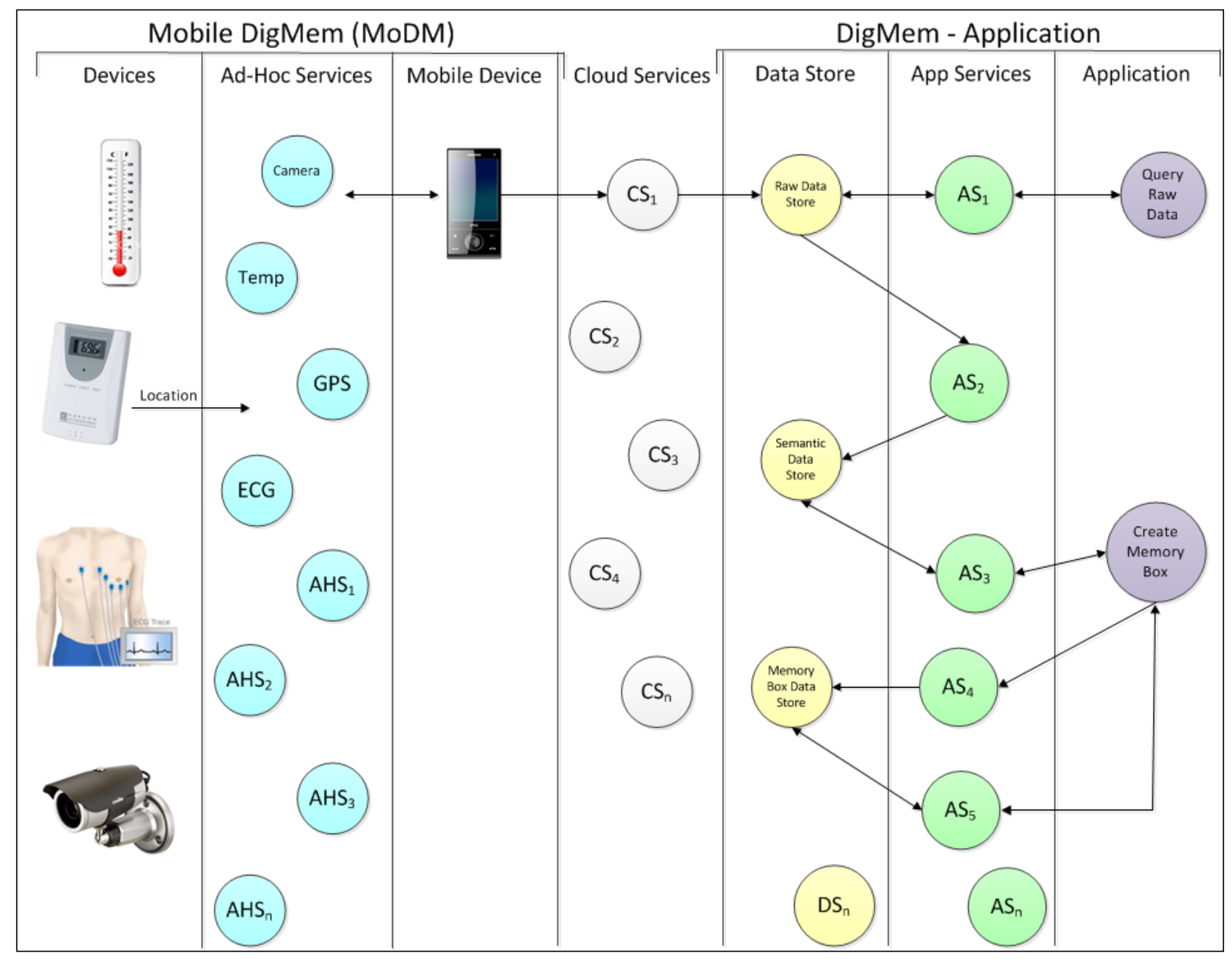

Figure 1. DigMem System Design

The data is then transferred to the DigMem portion of the system, using cloud computing technologies, where it is transformed into a "memory box". The raw data is transformed into the Resource Description Framework (RDF) specification and stored within the Semantic Data Store. Converting the data into this format allows structured and semi-structured data to be mixed, exposed, and shared across different applications [30]. Therefore, links between numerous data items can be established and the memories created will be a "mash-up" of a broad range of data. The data that we are able to include in these memories is not limited to one specific type. This is a unique aspect of the system because any data source can be added to our memory, irrespective of its data type. This information is then queried, and a memory box is created.

Once a memory box has been created the queries will then be stored within the "Memory Box Data Store". These queries will be able to be retrieved, and memories can be reflected upon. These memories can be linked, forming a continuous stream of data. As the data sources, which we have access to, change so will the memories that are being created. Therefore, no two memories will be same. The ability to create memories that are reflective of our current environment is one novel aspect of the system. The following section outlines the DigMem system that is used to create these "boxes".

\section{A. Technical Details}

The prototype uses a Samsung Galaxy tablet and NeXus10 body sensors [31] (see Fig. 2) to gather location, photo and physiological (ECG) information, about the user's journeys. Below, Figure 3 a) illustrates the wearable body sensors, attached to the user, whilst, Figure $3 \mathrm{~b}$ ) depicts all the mobile devices that were used in the demonstration, to gather information.

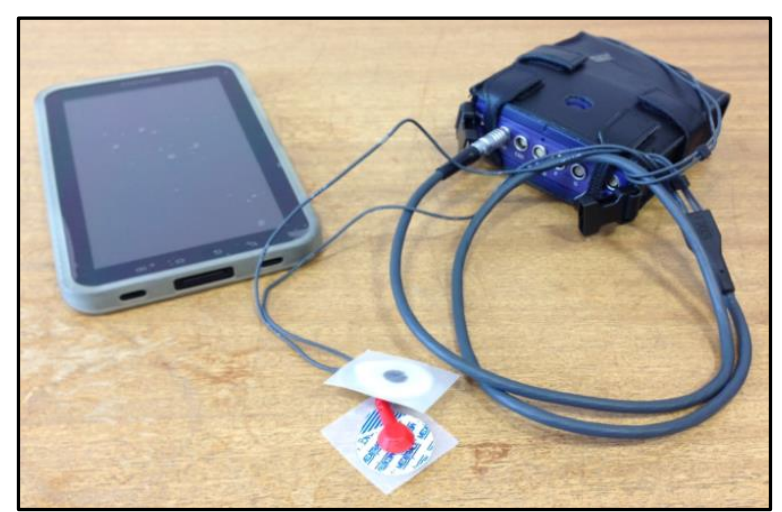

Figure 2. Equipment used to collect DigMem information 


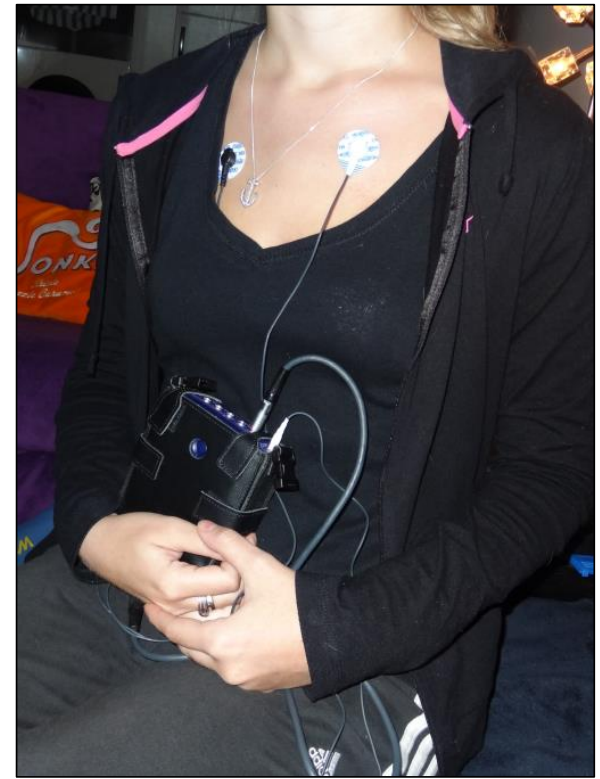

(a)

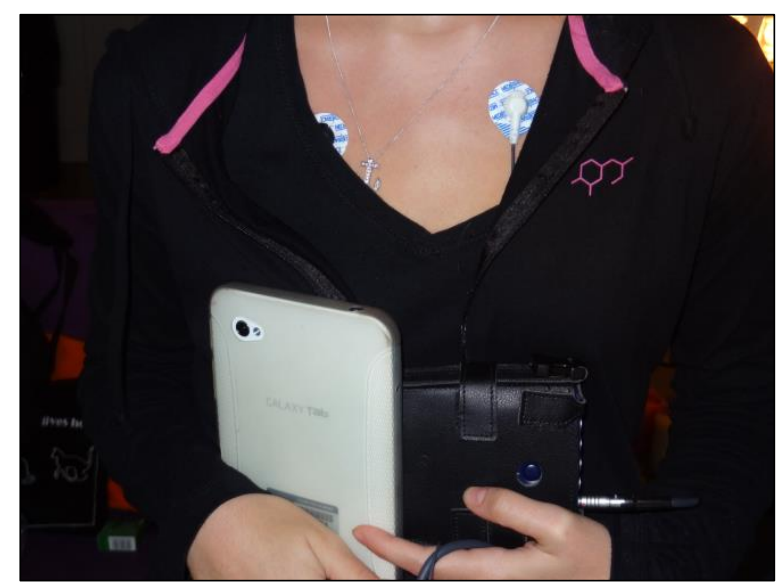

(b)

Figure 3. Wearable body sensors (a) and setup used to gather data (b)

Using this set up, the system is capable of collecting these various pieces of information, throughout the day. Since GPS is limited to outside environments, the prototype has only been deployed when the user is going into this setting. For example, in going to work or walking. Photo and GPS services are ran on the tablet and the body sensors transmit their readings to a laptop, via Bluetooth.

The data is stored in the user's DropBox [32] folder, located locally on the laptop and tablet devices. Once these devices are connected to $\mathrm{Wi}-\mathrm{Fi}$, the data is automatically uploaded to the cloud. A number of Python scripts are then run, which checks the DropBox folder and moves the information into the Raw Data Store. This store is a MySQL database, which is located on a server within Liverpool John Moores University (LJMU).

Once the raw data is stored another script is run that creates RDF documents of the data. The RDF data is then stored in the Semantic Data Store. A web interface has been developed, which displays the memory boxes and provides a way to query the information. In order to create the boxes, SPARQL queries are performed on the RDF data. The interface uses a SPARQL endpoint (see Fig. 4) to enable the execution of the queries, over the web. The endpoint has been implemented using ARC2 [33]. This method allows information, from any data source, to be used in the memory.

Once a query has been executed, the results are transformed into the JavaScript Object Notation (JSON) format and displayed as a memory box. The query "Where was I, what was I doing and how did I feel on $17^{\text {th }}$ July 2012 at 8:35pm?" has been transformed into a memory box (see Fig. 5). This setup enables the raw data to be transformed into visual items and allows the user to interpret their physiological readings.

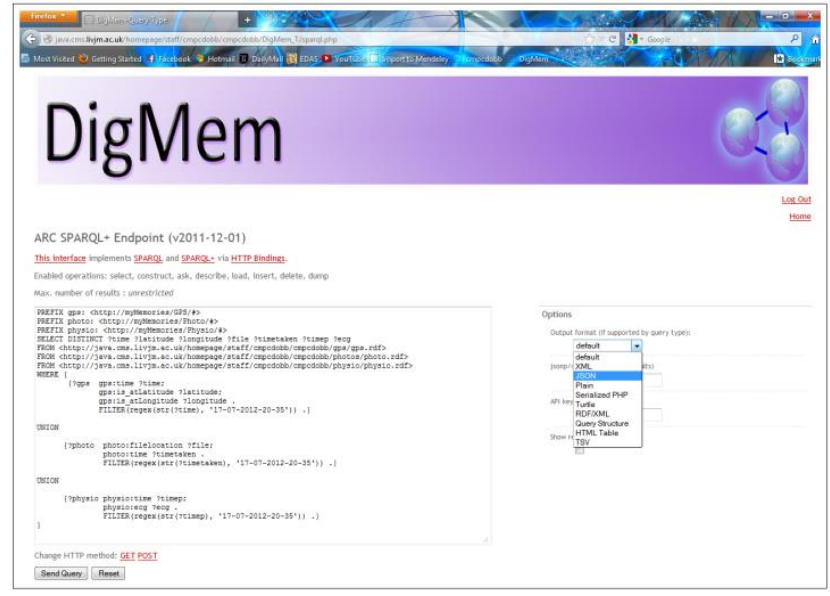

Figure 4. DigMem Web Interface with SPARQL Endpoint
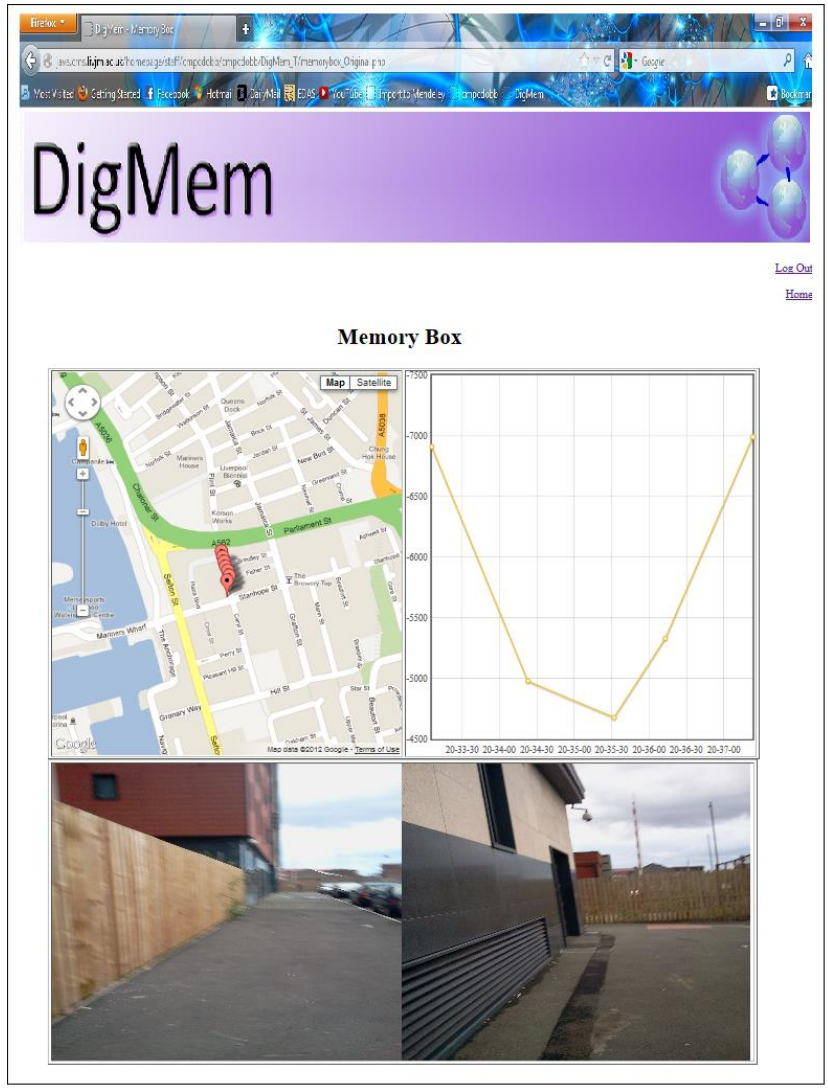

Figure 5. DigMem Memory Box 
DigMem offers users the ability to bring together a variety of information, reason over this and illustrate where we were and what our bodies were going through at a particular time. Any number of services can be incorporated, such as temperature, other people and even building information. The system has the ability to create a more vivid and dynamic memory than previously seen.

\section{B. Discussion}

As it can be seen from the demonstration, a successfully created memory box has been created and augmented with physiological data. The contents, from the memory box, indicate where the user was, what they were doing and what their body was going through. It can be concluded that on the $17^{\text {th }}$ July 2012 , at $8: 35 \mathrm{pm}$, the user was walking around Liverpool. The results also indicate that they may have slowed down or taken a break, as their heart rate had dropped slightly during their walk.

Although a limited use of the system has been presented, a great deal can still be learnt from this development. DigMem illustrates that by augmenting HDMs with physiological data a greater level of detail is incorporated into the memory. This is important because it provides a richer understanding about aspects of our health, level of activity and physical wellbeing. This information can be reasoned over and questions about ourselves can be queried, such as, "How was I feeling at $x$ point in my life?" "What factors made me feel like this?" and "How were others around me feeling at the same time?" The use of linked data makes these queries possible to execute. This is because data, from any device, can be brought together and used in the HDM. Any time of our lives can be reconstructed and our feelings, from those times, reasoned over.

There are several notable features that make this system a viable option for creating HDMs. In particular, the use of body sensors enables a greater level of detail to be incorporated into the memory. We can visually see how our bodies have changed over the years and what factors contributed to those changes. Secondly, by using ubiquitous devices memory data can be collected from a vast array of items. Any device that is running the MoDM middleware platform can be used in the memory. There is no need to buy specialist equipment, such as a SenseCam, to collect data. At the very least, memories can be created using only a mobile phone. The use of ubiquitous devices also allows unique memories to be created. A memory created in one environment will have access to distinct data sources that might not be present in other. Therefore, as we move through different environments, our data sources will change and the memories created will be unique.

This framework provides a flexible solution for creating HDMs, in any environment. The user is able to keep a record of their entire life and reflect upon any moment. Physiological data allows us to see exactly how our bodies react in certain situations. This has the ability to influence almost every aspect of our lives, particularly our health. Prolonged periods of sedentary behaviour could be identified, which could establish how our bodies were affected. The effect of behavioural changes, such as walking more, could also be projected to provide a better incentive for change.

\section{CONCLUSIONS AND FUTURE WORK}

Physiological monitoring allows us to measure biological responses and is a powerful tool for quantifying behaviour. A direct correlation between certain stimuli and physiological responses can be ascertained. By incorporating this data into our HDMs a more dynamic representation of our memories can be built. The DigMem system provides a flexible method of capturing and creating memories. A lifetime of data can be reasoned over and behaviour can be reasoned over. Digital memories will grow alongside their human counterparts.

Future work aims to build on the ideas presented so that a catalogue of memories can be accumulated. Memory boxes themselves are temporal. Therefore, the queries that are used to create these memories will need to be archived. Future work aims to focus on creating this archive and extending the system so that past queries can be searched through and retrieved. In this sense, a catalogue of our lives will begin to emerge, which can be reasoned over.

Long-term monitoring of participants is also another area that needs further investigation. Studying participants for an extended amount of time has the potential to, as stated above, discover how certain stimuli may influence behaviour. For example, we might be more inactive in the winter, due to a drop in temperature, or during the Olympics, more sport was being played. Using these collected memories, our health can also be monitored [34-37]. Markers for an increase in sedentary behaviour can be identified. HDMs provide the visual evidence needed to depict how behaviour can influence certain aspects of our lives. However, due to the cumbersome and intrusive nature of sensing devices, longterm monitoring is quite difficult. Nevertheless, as technology develops these devices will become smaller, more convenient and more common, in everyday life. Sensors embedded in ordinary objects, and even in ourselves, will be able to transmit information, which can be incorporated into HDMs. This work paves the way, and provides a method, for creating HDMs over a lifetime. As devices and technology advances, DigMem can accommodate these changes and a true reflection of our lives will begin to emerge.

\section{REFERENCES}

[1] A. R. Doherty, "Human Digital Memories," Health Promotion, no. July, 2005.

[2] A. Fitzgibbon and E. Reiter, "Grand Challenges in Computing Research: GC3 Memories for life: managing information over a human lifetime," Jan. 2005.

[3] K. Gilleade and S. H. Fairclough, "Physiology as XP Body Blogging to Victory," in BioS-Play Workshop at Fun and Games, Leuven, Belgium, 2010.

[4] M. Weiser, "The Computer for the 21st Century," ACM SIGMOBILE Mobile Computing and Communications Review, vol. 3, no. 3, pp. 3-11, Jul. 1999.

[5] A. R. Doherty, N. Caprani, C. Ó. Conaire, V. Kalnikaite, C. Gurrin, A. F. Smeaton, and N. E. O'Connor, "Passively Recognising Human Activities Through Lifelogging," Computers in Human Behavior, vol. 27, no. 5, pp. 1948 1958, Sep. 2011. 
C. Dobbins, M. Merabti, P. Fergus, and D. Llewellyn-Jones, “Augmenting Human Digital Memories with Physiological Data," in The 3rd IEEE International Conference on Network Embedded Systems for Every Application (NESEA'12), 2012, pp. 35-41.

[6] S. Hodges, L. Williams, E. Berry, S. Izadi, J. Srinivasan, A. Butler, G. Smyth, N. Kapur, and K. Wood, "SenseCam: A Retrospective Memory Aid," UbiComp 2006: Ubiquitous Computing, vol. 4206, pp. 177-193, 2006.

[7] H. Lee, A. F. Smeaton, N. E. O'Connor, G. Jones, M. Blighe, D. Byrne, A. Doherty, and C. Gurrin, "Constructing a SenseCam Visual Diary As A Media Process," Multimedia Systems, vol. 14, no. 6, pp. 341349, Jul. 2008.

[8] A. R. Doherty, C. J. A. Moulin, and A. F. Smeaton, "Automatically assisting human memory: A SenseCam browser," Memory, pp. 1-11, Sep. 2010.

[9] A. Sellen, A. Fogg, M. Aitken, S. Hodges, C. Rother, and K. Wood, "Do Life-Logging Technologies Support Memory for the Past? An Experimental Study Using SenseCam," Conference on Human Factors in Computing Systems, CHI '07, Irvine, CA, (2007), pp. 8190, 2007.

[10] S. E. Lindley, D. Randall, W. Sharrock, M. Glancy, N. Smyth, and R. Harper, "Narrative, Memory and Practice: Tensions and Choices in the Use of a Digital Artefact," Proceedings of the 23rd British HCI Group Annual Conference on People and Computers: Celebrating People and Technology, pp. 1-9, Sep. 2009.

[11] V. Kalnikaite, A. Sellen, S. Whittaker, and D. Kirk, "Now Let Me See Where I Was: Understanding How Lifelogs Mediate Memory," Proceedings of the 28th International Conference on Human Factors in Computing Systems CHI '10, p. 2045, Apr. 2010.

[12] P. Kelly, A. Doherty, E. Berry, S. Hodges, A. M. Batterham, and C. Foster, "Can We Use Digital Life-Log Images To Investigate Active And Sedentary Travel Behaviour? Results From A Pilot Study," The International Journal of Behavioral Nutrition and Physical Activity, vol. 8, no. 1, p. 44, Jan. 2011.

[13] D. Byrne and G. J. F. Jones, "Towards Computational Autobiographical Narratives through Human Digital Memories," Proceeding of the 2nd ACM international workshop on Story representation, mechanism and context - SRMC '08, p. 9, Oct. 2008.

[14] B. Kikhia, J. Hallberg, J. E. Bengtsson, S. Sävenstedt, and K. Synnes, "Building Digital Life Stories For Memory Support," International Journal of Computers in Healthcare, vol. 1, no. 2, pp. 161-176, 2010.

[15] J. Gemmell, G. Bell, R. Lueder, S. Drucker, and C. Wong, "MyLifeBits: Fulfilling the Memex Vision," International Multimedia Conference, p. 235, 2002.

[16] BodyMedia Inc., "SenseWear: Introducing the Enhanced BodyMedia SenseWear System," 2011. [Online]. Available: http://sensewear.bodymedia.com/SW-LearnMore/Product-Overview.

[17] T. J. Dwyer, J. A. Alison, Z. J. McKeough, M. R. Elkins, and P. T. P. Bye, "Evaluation of the SenseWear activity monitor during exercise in cystic fibrosis and in health.," Respiratory Medicine, vol. 103, no. 10, pp. 1511-1517, Oct. 2009.

[18] G. J. M. Almeida, M. C. M. Wasko, K. Jeong, C. G. Moore, and S. R. Piva, "Physical Activity Measured by the SenseWear Armband in Women With Rheumatoid Arthritis," Physical Therapy: Journal of the American Physical Therapy Association, Jun. 2011.

[19] R. Paradiso, G. Loriga, and N. Taccini, "A Wearable Health Care System Based on Knitted Integrated Sensors," IEEE Transactions on Information Technology in Biomedicine, vol. 9, no. 3, pp. 337-344, Sep. 2005.
[20] K. Taraldsen, T. Askim, O. Sletvold, E. K. Einarsen, K. G. Bjåstad, B. Indredavik, and J. L. Helbostad, "Evaluation of a Body-Worn Sensor System to Measure Physical Activity in Older People With Impaired Function," Physical Therapy: Journal of the American Physical Therapy Association, vol. 91, no. 2, pp. 277 285, Feb. 2011.

[21] G. López, V. Custodio, and J. I. Moreno, "LOBIN: Etextile and wireless-sensor-network-based platform for healthcare monitoring in future hospital environments," IEEE Transactions on Information Technology in Biomedicine, vol. 14, no. 6, pp. 1446-1458, Nov. 2010.

[22] C. P. Figueiredo, K. Becher, K. P. Hoffmann, and P. M. Mendes, "Low Power Wireless Acquisition Module for Wearable Health Monitoring Systems," in 32nd Annual International Conference of the IEEE Engineering in Medicine and Biology Society (EMBC), 2010, pp. 704 707.

[23] A. Pantelopoulos and N. G. Bourbakis, "Prognosis - A Wearable Health-Monitoring System for People at Risk: Methodology and Modeling," IEEE Transactions on Information Technology in Biomedicine, vol. 14, no. 3, pp. 613-21, May 2010.

[24] M. Pacelli, G. Loriga, N. Taccini, and R. Paradiso, "Sensing Fabrics for Monitoring Physiological and Biomechanical Variables: E-textile solutions," in 3rd IEEE-EMBS International Summer School and Symposium on Medical Devices and Biosensors, 2006, pp. 1-4

[25] P. S. Pandian, K. Mohanavelu, K. P. Safeer, T. M. Kotresh, D. T. Shakunthala, P. Gopal, and V. C. Padaki, "Smart Vest: Wearable Multi-Parameter Remote Physiological Monitoring System," Medical engineering \& physics, vol. 30, no. 4, pp. 466-77, May 2008.

[26] S. Coyle, K.-T. Lau, N. Moyna, D. O'Gorman, D. Diamond, F. Di Francesco, D. Costanzo, P. Salvo, M. G. Trivella, D. E. De Rossi, N. Taccini, R. Paradiso, J.-A. Porchet, A. Ridolfi, J. Luprano, C. Chuzel, T. Lanier, F. Revol-Cavalier, S. Schoumacker, V. Mourier, I. Chartier, R. Convert, H. De-Moncuit, and C. Bini, "BIOTEXBiosensing Textiles for Personalised Healthcare Management," IEEE Transactions on Information Technology in Biomedicine, vol. 14, no. 2, pp. 364-370, Mar. 2010.

[27] D. Curone, E. L. Secco, A. Tognetti, G. Loriga, G. Dudnik, M. Risatti, R. Whyte, A. Bonfiglio, and G. Magenes, "Smart Garments for Emergency Operators: The ProeTEX Project," IEEE Transactions on Information Technology in Biomedicine, vol. 14, no. 3, pp. 694-701, May 2010

[28] G. Langereis, L. de Voogd-Claessen, A. Spaepen, A Sipliä, C. Rotsch, and T. Linz, "ConText: Contactless sensors for body monitoring incorporated in textiles," in IEEE International Conference on Portable Information Devices, 2007, vol. 027291, pp. 1-5.

[29] R. Matthews, N. J. McDonald, P. Hervieux, P. J. Turner, and M. A. Steindorf, "A wearable physiological sensor suite for unobtrusive monitoring of physiological and cognitive state," in 29th Annual International Conference of the IEEE Engineering in Medicine and Biology Society (EMBS), 2007, pp. 5276-5281.

[30] W3C, "RDF," 2004. [Online]. Available: http://www.w3.org/RDF/.

[31] Mind Media BV, "About NeXus Technology." [Online]. Available:

http://www.mindmedia.nl/CMS/en/applications/research -a-physiology/about-nexus-technology.html. 
C. Dobbins, M. Merabti, P. Fergus, and D. Llewellyn-Jones, "Augmenting Human Digital Memories with Physiological Data," in The 3rd IEEE International Conference on Network Embedded Systems for Every Application (NESEA'12), 2012, pp. 35-41.

[32] DropBox.com, "What is Dropbox ? The story of Dropbox How Dropbox works Features." [Online]. Available: http://www.dropbox.com/static/docs/DropboxFactSheet. pdf.

[33] semsol / Benjamin Nowack, "Easy RDF and SPARQL for LAMP Systems," 2012. [Online]. Available: https://github.com/semsol/arc2/wiki.

[34] C. Dobbins, P. Fergus, M. Merabti, and D. LlewellynJones, "Monitoring and Measuring Sedentary Behaviour with the Aid of Human Digital Memories," in Proceedings of The 9th Annual IEEE Consumer Communications \& Networking Conference (CCNC'12), 2nd IEEE International Workshop on Consumer eHealth Platforms, Services and Applications (CeHPSA), Las Vegas, NV, USA, 2012, pp. 395-398.

[35] C. Dobbins, P. Fergus, M. Merabti, and D. LlewellynJones, "Remotely Monitoring and Preventing the Development of Pressure Ulcers with the Aid of Human
Digital Memories," in Proceedings of 47th Annual IEEE International Conference on Communications (ICC'12), 1st IEEE International Workshop on Mobile Consumer Health Care Networks, Systems and Services (MobiCHeSS), Ottawa, Canada, 2012, pp. 7669-7673.

[36] C. Dobbins, P. Fergus, G. Stratton, M. Rosenberg, and M. Merabti, "Monitoring and Reducing Sedentary Behaviour in the Elderly with the Aid of Human Digital Memories," Telemedicine and e-Health Journal, in press, vol. 19, no. 3, 2013.

[37] P. Fergus, A. Attwood, C. Dobbins, G. Stratton, A. Hussain, D. Al-jumeily, and M. Randles, "Monitoring and Measuring Physical Activity and Sedentary Behaviour," International Journal of Healthcare Technology and Management (IJHTM), in press, 2012. 